\title{
Intervenção precoce no enfarte da relação
}

José Agostinho Santos*

\section{RESUMO}

Introdução: O doente coronário tem procurado o seu médico de família (MF) em busca de aconselhamento relativo à actividade sexual (AS). Porém, até hoje, a abordagem desta tem permanecido algo deficitária.

Descrição do caso: Rui, 56 anos, antecedentes de angina estável classe I. Em Março/2010, na primeira consulta com actual MF, nega queixas, porém a esposa documenta irritabilidade desde 2009, data do diagnóstico da doença coronária. Em Setembro/2010, a esposa refere um agravamento da irritabilidade de Rui, com isolamento social progressivo e prejuízo na relação conjugal. Em Outubro/2010, Rui confirma que esta alteração comportamental coincidiu com o diagnóstico da cardiopatia isquémica. Faz-se um aconselhamento para a prática de exercício, actividade intraconjugal de lazer e sexual e retoma do convívio social. Uma semana depois, volta por dúvidas relativamente às actividades a praticar, questionando «mesmo a actividade sexual?!». Diz que o seu médico anterior o teria informado que possuía um risco aumentado de enfarte agudo do miocárdio (EAM) associado à AS e recomendado «ter cuidado!». Uma intervenção precoce é iniciada, explicando o baixo risco absoluto de EAM associado ao acto sexual e reiterando a segurança da AS num doente com angina estável classe I. O casal volta posteriormente, permitindo o reforço da confiança na sua AS.

Comentário: O impacto da cardiopatia isquémica na AS é compreendido pela diminuição da auto-estima e pelos efeitos laterais da farmacoterapia. $O$ aconselhamento constitui importante parte da terapêutica não-farmacológica. Se desadequado, pode ser iatrogénico. Constituindo a sexualidade uma artéria de suprimento afectivo num casal, um aconselhamento inseguro foi o trigger de um processo bloqueador deste suprimento e, assim, de um enfarte da relação conjugal. Uma trombólise foi realizada para eliminar o trombo de receios. A revascularização foi conseguida posteriormente, intervindo-se no casal com a doença afectiva coronária.

Palavras chave: Actividade Sexual; Doença Coronária; Aconselhamento.

\section{INTRODUÇÃO}

$\wedge$ s estratégias de prevenção secundária têm conseguido que o impacto das doenças cardiovasculares seja cada vez menor na capacidade funcional do doente. Este tem procurado o seu médico em busca de aconselhamento relativo a actividades da vida diária, incluindo a actividade sexual. ${ }^{1,2}$ No entanto, as dúvidas sobre a segurança da actividade sexual num doente com cardiopatia isquémica são tão comuns nele próprio como no seu médico. Até aos dias de hoje a abordagem da actividade sexual tem permanecido algo deficitária, quer em consultas de rotina quer no dia da alta hospitalar após um evento cardiovascular, o que tem precipitado uma crescente insegurança e desinformação do médico. ${ }^{1,3}$ Tal foi fru-

*Interno de Medicina Geral e Familiar, USF Lagoa, Centro de Saúde da Senhora da Hora, ULS - Matosinhos. to de um tabu transgeracional associado à sexualidade que agora tende a apagar-se. ${ }^{1}$

Este caso clínico dá relevância ao aconselhamento médico. Fazendo parte do tratamento não-farmacológico, pode ser altamente terapêutico, pouco dispendioso, acessível e ter grande impacto na orientação de qualquer doente, neste caso em particular, do paciente coronário. Porém, tal como uma farmacoterapia inadequada, um aconselhamento inseguro poderá também ter efeitos laterais indesejáveis.

\section{DESCRIÇÃO DO CASO}

\section{Dados demográficos}

Rui (nome fictício) é um homem de 56 anos, engenheiro, natural de Vila Real e actualmente residente na Senhora da Hora. É o mais novo de três irmãos. Actualmente, casado com Maria (nome fictício). O casal insere-se numa família nuclear, na faseVI do ciclo de Duvall. 
Do seu genograma (Figura 1), realçam-se os seguintes antecedentes familiares: a mãe com Diabetes Mellitus tipo 2 e Hipertensão Arterial e o irmão mais velho com Hipertensão Arterial.

\section{História Médica Pregressa}

Como antecedentes pessoais activos, Rui tem

- doença coronária diagnosticada em 2009, após um quadro sugestivo de angina estável classe I (Canadian Cardiovascular Society, CCS);

- hipertensão arterial essencial grau 1 (European Society of Cardiology/European Society of Hypertension) desde 2000;

- excesso ponderal.

A medicação habitual inclui lisinopril 20 mg + hidroclorotiazida 12,5 mg/dia, ácido acetilsalicílico 150 $\mathrm{mg} /$ dia, atenolol $50 \mathrm{mg} / \mathrm{dia}$, rosuvastatina $10 \mathrm{mg} / \mathrm{dia}$ e nitroglicerina $0,5 \mathrm{mg}$ sublingual em SOS (dor anginosa).

É ex-fumador (carga tabágica de 10 UMA durante 20 anos) e ingere 19,2 g de álcool por dia. No entanto, revela ter tido hábitos semanais de consumo excessivo de bebidas destiladas em eventos sociais, até meados de 2009.

Praticou futebol num clube regional dos 13 aos 25 anos. Desde há cerca de seis meses faz uma caminhada nocturna durante 30 a 60 minutos.

A História da Doença Actual é, abaixo, dividida segundo as datas das consultas. O raciocínio clínico desenvolvido em cada contacto com o paciente é parte integrante de cada divisão.

\section{2/Março/2010}

Vem à sua primeira consulta com a actual médica de família (MF), juntamente com sua esposa Maria. Ambos entraram recentemente para a lista de utentes, após mudança de centro de saúde e de zona residencial. O motivo desta consulta consiste em estabelecer o contacto com a nova MF e actualizar os seus perfis de saúde. É portador dos exames complementares mais recentes.

A nível subjectivo, Rui nega queixas. É questionado sobre o seu estado geral de saúde, respondendo «estou bem dentro do possível... enquanto cá se vai andando, é bom...» (sic). A esposa interrompe a entrevista clínica e mostra discordância. Refere que Rui tem andado mais ansioso e facilmente irritável desde meados de 2009, altura do diagnóstico da cardiopatia isquémica, e verbaliza «enerva-se com tudo e está sempre a descarregar em cima de mim...» (sic).

Rui acaba por confirmar que anda mais irritável, porém atribui tal alteração do comportamento à sua recente sobrecarga laboral. Nega perturbações do sono ou da memória. Nega sentir-se triste e desmotivado para actividades de lazer (leitura e fotografia). Iniciou novo hobbie, a pesca. Porém, abandonou um passatempo antigo: jogar xadrez com os amigos.

Sob o ponto de vista cardiovascular, encontra-se sem dor e refere nunca ter tido necessidade de recorrer à nitroglicerina, nem mesmo durante a sua caminhada diária. É inquirido sobre a história anterior que conduziu ao diagnóstico de cardiopatia isquémica. Rui documenta que em Junho/2009, após 5 minutos de jogo de futebol com amigos, desenvolveu uma dor retroesternal tipo peso, intensa, com irradiação para a metade esquerda do pescoço e que teve alívio após 5 minutos de repouso. Recorreu ao seu anterior médico de família, pelo que foi pedido um estudo que incluiu prova de esforço electrocardiográfica.

A nível objectivo nesta consulta, realça-se um aspecto cuidado e idade aparente correspondente à idade real. Apresenta TA = 124/78 $\mathrm{mmHg}$, peso $=94,2 \mathrm{Kg}$, altura $=1,83 \mathrm{~m}$, IMC $=28,1 \mathrm{Kg} / \mathrm{m}^{2}$ e perímetro abdominal $=91 \mathrm{~cm}$. A auscultação cardiopulmonar é normal. Sem alterações relevantes no restante exame físico.

Os exames complementares revelam um colesterol total $=182 \mathrm{mg} / \mathrm{dL}$, $\mathrm{HDL}=65 \mathrm{mg} / \mathrm{dL}, \mathrm{LDL}=88 \mathrm{mg} / \mathrm{dL}$, triglicerídeos = $143 \mathrm{mg} / \mathrm{dL}$ e hemograma

Figura 1. Genograma familiar. 
e perfis renal, hepático, tiroideu sem alterações. Traz também os registos dos exames complementares de diagnóstico solicitados pelo seu anterior médico de família. O Rx Tórax e o ECG (Junho/2009) são normais, o ecocardiograma (Junho/2009) revela uma hipertrofia ventricular esquerda e o relatório da prova de esforço electrocardiográfica (Junho/2009) indica uma presumível doença coronária aterosclerótica: «prova interrompida aos $10 \mathrm{~min}$ e 1 seg (estádio 4 do protocolo de Bruce; 11 METs) por dor retroesternal moderada coincidente com um infradesnivelamento de ST de $1 \mathrm{~mm}$ em DII, DIII e aVF».

Uma vez que o paciente se encontra com bom controlo sintomático, metabólico e tensional, o plano inclui a manutenção de atitudes e marcação de nova consulta entre três a seis meses.

\section{2/Setembro/2010}

O casal marca consulta seis meses depois. No entanto, neste dia da consulta, apenas vem a esposa e Rui falta por motivos profissionais. Maria refere preocupação com o comportamento de Rui, que continua irritável e progressivamente mais isolado, refugiando-se nas suas actividades de lazer solitárias. Conta que esta alteração comportamental causa dano na relação conjugal: «já nem falamos muito, que é para não nos chatearmos...», «agora já nem vai aos jantares com os amigos...», «nunca mais conseguiu ultrapassar o problema do coração... e além disso, não pára de trabalhar...» (sic). É marcada nova consulta para Rui dentro de um mês, altura mais conveniente para o utente.

\section{2/Outubro/2010}

No mês seguinte, o doente vem sozinho à consulta e refere estar «saturado» da actividade profissional e de lidar com colegas de trabalho. Nega mau ambiente no local de trabalho ou problemas financeiros da empresa assim como humor deprimido, apatia ou anedonia. Contudo, documenta ele próprio um isolamento social progressivo: «ando mais neura... por isso, quero estar no meu canto e mantenho-me activo a fazer as coisas que gosto...» (sic).

Quando questionado, revela que esta alteração de comportamento teve início insidioso com o diagnóstico de doença coronária. Torna-se perceptível que este diagnóstico abalou a auto-estima de Rui quando diz: «mexeu um bocado comigo... sempre me vi como um homem de ferro... até pratiquei futebol durante 12 anos!». Não esconde que tal comportamento e isolamento progressivo estejam a corroer a relação marital.

Parece claro à sua MF que este homem apresenta alguns sintomas depressivos, embora seja questionável se preenche o número suficiente de critérios para um diagnóstico de perturbação depressiva major. Estes sintomas depressivos são interpretados num contexto dependente do diagnóstico de doença coronária em meados de 2009, ao qual se associa um luto por perda da auto-imagem de pessoa saudável ou um rótulo que, aparentemente, frustra um homem que foi futebolista e que é um engenheiro com uma preenchida vida de relação.

Explicam-se, então, a etiologia e as diferentes classes da sua patologia cardíaca. Esclarece-se que a sua classificação funcional (classe I-CCS) lhe permite continuar a desfrutar de inúmeros prazeres da sua vida e faz-se um aconselhamento genérico relativo a estilos de vida, sob o ponto de vista biopsicossocial. Sob o prisma biológico, é recomendado a privilegiar uma alimentação mediterrânica e a manter o seu exercício aeróbico (caminhada). A nível psicológico, aconselha-se que mantenha a busca de prazer em actividades de lazer, que perpetue os circuitos familiares de afecto e que procure retirar bons momentos de intimidade com a sua esposa, nomeadamente através da actividade sexual. E a nível social, sugere-se que recupere a interacção com os seus amigos, da qual retire vivências que possam ser, de algum modo, enriquecedoras.

Como parte do plano, marca-se nova consulta para o mês seguinte para reavaliação.

\section{9/Outubro/2010}

Rui vem à consulta aberta na semana seguinte, referindo sentir-se «obsessivo» e com dificuldade de concentração no trabalho. Com o adiantar da entrevista clínica, revela que relaciona o aumento da sua ansiedade basal com a preocupação relativa a actividades que possa desempenhar no seu quotidiano. E posteriormente, questiona: «mesmo a actividade sexual?!»

É reforçada a resposta positiva, de acordo com o aconselhamento elaborado na consulta anterior. Rui afirma, então, que o seu médico assistente anterior o terá informado, perante o diagnóstico da doença coronária, que possuía um risco aumentado de enfarte agudo do miocárdio associado a relações sexuais e que o terá aconselhado a «ter cuidado» (sic). 
O paciente refere que «não conseguirei ter relações a pensar que posso ter um enfarte... não sei bem como se tem cuidado durante as relações... nunca mais tive uma relação sexual..."

Nesta consulta aberta entende-se o que perturba este paciente, desvenda-se a causa daquela alteração insidiosa do seu comportamento e compreende-se o dano prolongado na sua relação conjugal. Uma analogia oportuna é possível ser elaborada durante o raciocínio médico: tal como a artéria coronária é responsável pelo suprimento sanguíneo para o coração, a actividade sexual constitui uma importante artéria de suprimento afectivo para a relação conjugal. O diagnóstico da doença coronária constitui, por si só, um peso que comprime a auto-estima, prejudica a performance sexual e, tal como uma placa de ateroma, estenosa esta artéria da sexualidade. A recepção do aconselhamento pelo seu anterior médico funcionou como uma terapêutica não-farmacológica desadequada, criando o medo no paciente, e foi o trigger para a ruptura da placa do diagnóstico que já estenosava a artéria da actividade sexual. Após esta ruptura criou-se, ao longo do tempo, um trombo de mitos, receios, ansiedade e frustração. Este trombo esteve, por sua vez, na origem do enfarte... da relação conjugal.

É retomado o aconselhamento elaborado na consulta anterior, explicando a relativa «benignidade» da classe I de angina estável para a prática da actividade sexual. É transmitido ao doente, utilizando uma linguagem ajustada, parte do conhecimento científico actual sobre a sexualidade no doente coronário.

É-lhe explicado que a actividade sexual apresenta um consumo energético médio de cerca de 3 a 5 METs (máximo na fase do orgasmo), um gasto metabólico semelhante ao da maioria das actividades do dia-a-dia, , ${ }^{1,2,4}$ nomeadamente similar à exigência cardíaca subjacente à sua caminhada nocturna que tolera bem (Quadro I).

Estudos de boa qualidade, nomeadamente case-crossover, foram realizados nos últimos anos envolvendo pacientes com doença coronária diagnosticada e sexualmente activos. Encontraram um risco relativo (RR) de $\mathrm{EAM} \approx 2,5$ para populações ocidentais ${ }^{5,6,7}(\approx 5,5$ para uma população latina-americana $)^{8}$ apenas nas duas horas após início da actividade sexual, comparativamente à não-prática de actividade sexual. No entanto, o risco absoluto permanece muito baixo.

Usando os dados do estudo de Framingham e um

\begin{tabular}{|c|c|}
\hline \multicolumn{2}{|c|}{$\begin{array}{l}\text { QUADRO I. Consumo energético estimado para } \\
\text { diferentes actividades }\end{array}$} \\
\hline Actividade & $\begin{array}{c}\text { Consumo } \\
\text { energético (METs) }\end{array}$ \\
\hline Caminhada a $3,5 \mathrm{Km} / \mathrm{h}$ & 2 \\
\hline $\begin{array}{l}\text { Actividade sexual pré-orga } \\
\text { intraconjugal }\end{array}$ & $2-3$ \\
\hline Caminhada a $5 \mathrm{Km} / \mathrm{h}$ & 3 \\
\hline $\begin{array}{l}\text { Actividade sexual orgasmo } \\
\text { intraconjugal (10-16 seg) }\end{array}$ & $4-5$ \\
\hline Subir 2 lances de escadas & 6 \\
\hline Corrida $10 \mathrm{Km} / \mathrm{h}$ & $6-7$ \\
\hline
\end{tabular}

factor de multiplicação = 2,5 (correspondente ao RR), $o$ acto sexual eleva o risco absoluto de EAM num homem de 50 anos (não-diabético e não-fumador) de 1 para 2,5 / 1000000 / hora. No homem de 50 anos com angina estável classe I eleva esse risco de 3 para 7,5 / 1000000 / hora e naquele pós-EAM de 10 para 25 / 1000000 / hora. Os aumentos do risco absoluto são, então, modestos e têm somente uma expressão nas duas horas após o acto sexual. Não se trata de um risco que permaneça durante os milhares de horas de vida do paciente. Este RR reduz-se em doentes coronários que praticam actividade física regular (como é o caso do Rui) para um valor muito próximo de $1 .{ }^{5,6}$ Por outro lado, a sua medicação habitual é, para além de antianginosa, preventiva de eventos cardiovasculares.

Apresentando estas três razões, pelas quais o paciente não tem contra-indicação para a prática de actividade sexual, é realizada uma intervenção precoce, cujo objectivo visa eliminar parte do trombo de mitos e receios e, assim, restituir a actividade funcional sexual. É, portanto, conseguida a trombólise nesta consulta.

\section{6/Outubro/2010}

Uma semana depois Rui volta à consulta com a esposa. Refere sentir-se «muito bem» e que retomou a actividade sexual «sem percalços!» (sic).

Maria revela-se «feliz», porém mantém alguma preocupação e pergunta «não haverá mesmo risco de causar um enfarte no meu marido durante as relações sexuais?»

Procurando dar a melhor resposta à questão, a evi- 
dência científica disponível sobre o tema é discutida com o casal. É explicado que um homem com 3 factores de risco (género, idade $>45$ anos e hipertenso) e angina estável classe I (SCC) se classifica como de «baixo risco», segundo as Normas do Segundo Consenso de Princeton. ${ }^{9}$ Este Consenso consiste num grupo de peritos cardiologistas que estabelece que, para a abordagem da sexualidade de um doente cardiovascular, deverá ser realizada uma rigorosa avaliação clínica. Os doentes de baixo risco (Quadro II), que representam a maioria dos doentes cardiovasculares, não apresentam qualquer contra-indicação para a prática da actividade sexual e devem ser encorajados a manter a sua actividade íntima intraconjugal. Os de risco intermédio deverão fazer uma estratificação não-invasiva (nomeadamente pela prova de esforço que documente a sua capacidade funcional), de maneira que possam ser classificados como baixo ou elevado risco. Apenas os de risco elevado deverão ter a sua actividade sexual deferida até o risco reduzir.

A prova de esforço electrocardiográfica, realizada pelo doente (apesar de este ser de baixo risco), constitui um importante factor mediador ao documentar que apenas com um exercício correspondente a 11 METs surge isquemia miocárdica, um gasto metabólico que representa o dobro do máximo exigido numa relação sexual intraconjugal. ${ }^{1,3} \mathrm{O}$ Rui apresenta, portanto, uma boa reserva funcional.

QUADRO II. Pacientes de baixo risco cardiovascular para a prática de actividade sexual, segundo as normas do segundo consenso de Princeton

\section{Pacientes de baixo risco cardiovascular}

- Assintomáticos

- < 3 factores de risco para doença arterial coronária (excepto género)

- Hipertensão arterial controlada

- Angina estável classe I-II (CCS)

- Pós-EAM submetido a revascularização miocárdica com sucesso

- Pós-EAM não complicado (> 8 semanas)

- Patologia valvular ligeira

- Insuficiência Cardíaca Estádio I (NYHA)
Com esta exposição da evidência actual, procedese, junto dos dois elementos da relação em risco, à eliminação total do trombo que obstruía parcialmente aquela artéria de suprimento afectivo. Permanecerá, embora de menor tamanho, uma placa ligeiramente obstrutiva do peso psicológico do diagnóstico da doença coronária, que provavelmente nunca será possível eliminar do seio daquele casal.

\section{Consultas seguintes}

Nas consultas seguintes os dois elementos do casal aparecem sempre juntos no gabinete. Rui encontra-se de humor claramente mais elevado e refere que retomou as actividades sociais, nomeadamente o xadrez com os amigos. Maria refere que «vivemos uma espécie de segunda lua-de-mel». Conclui-se, portanto, que foi conseguida com sucesso a revascularização da actividade sexual deste casal.

\section{COMENTÁRIO}

A actividade sexual constitui um aspecto importante do bem-estar biopsicossocial do indivíduo e, naturalmente, também o será para um doente com doença coronária.

O aconselhamento médico constitui parte significativa da terapêutica não-farmacológica e, quando integra insegurança e desinformação (por erro na sua transmissão ou na sua recepção), poderá ser desadequado e deletério. Neste caso em particular, originou um trombo no suprimento da afectividade deste casal e, consequentemente, um enfarte da relação conjugal.

Talvez porque a sexualidade constituiu um tabu na nossa comunidade, a sua abordagem foi deixada para segundo plano até aos dias de hoje. Tal perpetuou a desactualização e insegurança em parte da comunidade médica. ${ }^{1,3}$

Como comentário final, poder-se-á salientar a abordagem generalista dos estilos de vida, realizada na segunda consulta com o paciente. Tal aconselhamento terá tocado em diferentes aspectos de relevância segundo um modelo biopsicossocial, incluindo a actividade sexual. Acredita-se que tal abrangência terá permitido, por sua vez, abrir espaço para as dúvidas que levaram o paciente a revelar o que estaria na base da sua alteração de comportamento.

Por último, o facto do caso ter sido desenvolvido durante o seguimento por Medicina Geral e Familiar con- 
feriu um campo de actuação mais fácil, o que terá agilizado todo este processo bem sucedido de revascula rização. Na realidade, o Médico de Família tem a oportunidade de abordar diferentes elementos da família em diversos contactos, adquirindo informação adicional que lhe permitirá actuar de forma mais precoce em distintas situações clínicas. Neste caso em particular, as consultas com a esposa Maria permitiram percepcionar, de forma bem mais célere, que a afectividade daquele casal se encontrava em risco.

\section{AGRADECIMENTOS}

À Dra. Raquel Braga, à Dra. Mónica Granja e ao Dr. Luís Filipe Cavadas, pela amizade e pelo constante suporte científico e crítico

\section{REFERÊNCIAS BIBLIOGRÁFICAS}

1. Stein R, Hohmann CB. Atividade sexual e coração. Arq Bras Cardiol 2006 Jan; 86 (1): 61-7.

2. Cheitlin MD. Sexual activity and cardiac risk. Am J Cardiol 2005 Dec 26; 96 (12B): 24M-28M.

3. De Souza CA, Cardoso FL, Silveira RA, Martins C. Abordagem pelo cardiologista na actividade sexual do doente com doença arterial coronária. Acta Med Port 2011 Mar; 24 (2): 249-54.

4. Drory Y. Sexual activity and cardiovascular risk. Eur Heart J Supplements 2002 Dec; 4 (Suppl H): H13-H18.
5. Muller JE, Mittleman MA, Maclure M, Sherwood JB, Tofler GH. Triggering myocardial infarction by sexual activity: low absolute risk and prevention by regular physical exertion. JAMA 1996 May 8; 275 (18): 1405-9.

6. Möller J, Ahlbom A, Hulting J, Diderichsen F, de Faire U, Reuterwall C, et al. Sexual activity as a trigger of myocardial infarction: a case-crossover analysis in the Stockholm Heart Epidemiology Programme (SHEEP). Heart 2001 Oct; 86 (4): 387-90.

7. Dahabreh IJ, Paulus JK. Association of episodic physical and sexual activity with triggering of acute cardiac events: systematic review and metaanalysis. JAMA 2011 Mar 23; 305 (12): 1225-33.

8. Baylin A, Hernández-Díaz S, Siles X, Kabagambe EK, Campos H. Triggers of nonfatal myocardial infarction in Costa Rica: heavy physical exertion, sexual activity, and infection. Ann Epidemiol 2007 Feb; 17 (2): 112-8.

9. Kostis JB, Jackson G, Rosen R, Barrett-Connor E, Billups K, Burnett AL, et al. Sexual dysfunction and cardiac risk (the Second Princeton Consensus Conference). Am J Cardiol 2005 Jul 15; 96 (2): 313-21.

\section{CONFLITOS DE INTERESSES}

O autor declara ausência de conflitos de interesse.

\section{ENDEREÇO PARA CORRESPONDÊNCIA}

José Agostinho Santos

Rua Hernâni Torres, 73, 4. ${ }^{\circ}$ Dir

4200 - 320 Porto

E-mail: zeagostinho@hotmail.com

Recebido em 18/07/2011

Aceite para publicação em 24/11/2011

\section{ABSTRACT}

\section{EARLY INTERVENTION IN THE INFARCTION OF A RELATIONSHIP}

Introduction: Patients with coronary artery disease may seek advice from their general practitioner (GP) regarding sexual activity. However, this advice might sometimes be deficient.

Case Description: A 56 years-old man presented with a history of class I stable angina. In March, 2010, in his first consultation with his current GP, he had no complaints. His wife stated that he had been irritable since 2009, the date of the diagnosis of coronary artery disease. In September, 2010, his wife complained of his worsening of irritability with progressive social isolation and deterioration in the marital relationship. In October, 2010, he confirmed that this behavioral change coincided with the diagnosis of ischemic heart disease. He was counselled at this time regarding exercise, leisure, sexual activities and the resumption of his social life. A week later, he returned to the clinic with questions about permitted activity including sexual relations. He said that he has been told by a doctor in the past that he was at increased risk of acute myocardial infarction associated with sexual activity and had been told to "be careful". He then received an additional explanation of the low absolute risk of infarction associated with sex and reassurance regarding the safety of sexual activity in the class I angina patient. The couple returned to the clinic at subsequent visits reporting increased confidence in their sexual activity.

Comment: The impact of ischemic heart disease on sexual activity is believed to be due to both diminished self-esteem and the side effects of pharmacotherapy. Counseling is an important part of non-pharmacological therapy. Inadequate counselling can be harmful. Sexuality is compared to an artery serving as an emotional conduit for a couple. Ineffective counseling may be a trigger blocking this pathway causing infarction of the marital relationship. Counselling is like thrombolysis performed to eliminate the thrombus of fears. Revascularization of the relationship was achieved subsequently.

Keywords: Sexual Activity; Coronary Heart Disease; Counseling. 